
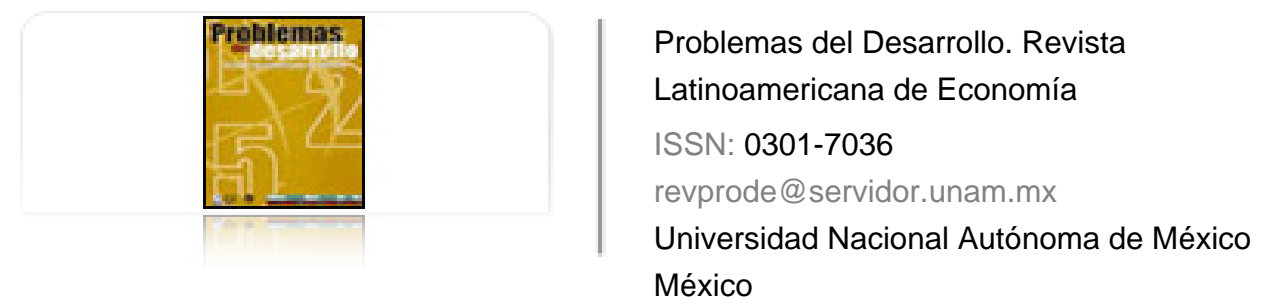

Coronel de Renolfi, Marta; Ortuño Pérez, Sigfredo Francisco Tipificación de los sistemas productivos agropecuarios en el área de riego de Santiago del Estero, Argentina

Problemas del Desarrollo. Revista Latinoamericana de Economía, vol. 36, núm. 140, 2005, pp. 63-88 Universidad Nacional Autónoma de México

Distrito Federal, México

Disponible en: http://www.redalyc.org/articulo.oa?id=11820092004

Cómo citar el artículo

- Número completo

- Más información del artículo

Página de la revista en redalyc.org

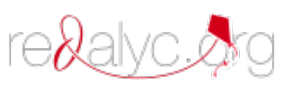

Sistema de Información Científica

Red de Revistas Científicas de América Latina, el Caribe, España y Portugal Proyecto académico sin fines de lucro, desarrollado bajo la iniciativa de acceso abierto 


\title{
TIPIFICACIÓN DE LOS SISTEMAS PRODUCTIVOS AGROPECUARIOS en el Área de riego de Santiago del Estero, Argentina
}

\section{Marta Coronel de Renolfi* Sigfredo Francisco Ortuño Pérez**}

Fecha de recepción: 2 de marzo de 2004. Fecha de aceptación: 2 de marzo de 2005.

\begin{abstract}
Resumen
El objetivo del trabajo es clasificar y tipificar una muestra de explotaciones rurales que pueda ser usada para la generación y transferencia de alternativas tecnológicas coherentes con las circunstancias de la agricultura de regadío en Santiago del Estero. La tipificación de los sistemas productivos permitió identificar formas de gestión rural bien diferenciadas entre sí, con necesidades y limitaciones diferentes, que facilitan la detección de debilidades y fortalezas, y establecer prioridades a la hora de diseñar políticas de desarrollo para esta zona.
\end{abstract}

Palabras clave: sistemas productivos, tipificación, análisis multivariante.

\begin{abstract}
The object of this study is to classify and typify a sample of rural operations to be used to generate and transfer coherent technological alternatives within the agricultural irrigation circumstances of Santiago del Estero. By typifying the productive systems it was possible to identify forms of rural management that are well differentiated among themselves, having different needs and limitations, making it possible to detect weaknesses and strengths, and establish priorities, while at the same time designing development policies for this zone.
\end{abstract}

Key terms: productive systems, typification, multivariate analysis.

* Profesora de Economía y Administración Forestal, Facultad de Ciencias Forestales, Universidad Nacional de Santiago del Estero, Argentina. Correo electrónico: mrenolfi@unse.edu.ar

* Profesor de economía rural, Universidad Politécnica de Madrid. Departamento de Economía y Gestión Forestal, Escuela Técnica Superior de Ingenieros de Montes, España. Correo electrónico: sortuno@montes.upm.es 


\section{Résumé}

L'objectif du travail est de classifier et de standardiser un échantillon d'exploitation rurale qui pourra être utilisé pour la génération et le transfert d'alternatives technologiques cohérentes avec les circonstances de l'agriculture irrigable à Santiago del Estero. La classification des systèmes productifs a permis d'identifier des formes de gestion rurale très différentes entre elles, avec des besoins et des limitations différentes, qui permettent de détecter des faiblesses et des points forts, ainsi que d'établir des priorités à l'heure de la conception des politiques de développement pour cette zone.

Mots clés: systèmes productifs, classification, analyse multi-variante.

\section{Resumo}

O objetivo do trabalho é classificar e tipificar uma mostra de explotações rurais que possa se usar para gerar e transferir alternativas tecnológicas coerentes com as circunstâncias da agricultura de regadio em Santiago del Estero. A tipificação dos sistemas produtivos permitiu a identificação de formas de gestão rural bem diferenciadas entre elas, com necessidades e limitações diferentes, que permitem detectar fraqueças e fortalezas, e estabelecer prioridades à hora de pensar em políticas de desenvolvimento para esta zona.

Palavras chave: sistemas produtivos, tipificação, análise multivariável. 


\section{Introducción}

$\mathrm{E}$

1 adecuado conocimiento de las circunstancias del productor rural es la base de

todo proceso de investigación y transferencia. La tecnología que se genere debe

ser elaborada a la medida de dichas circunstancias y de sus limitaciones y posibilidades. No es preciso argumentar la evidente conveniencia de diversificar la oferta tecnológica según tipos de fincas, pero es necesario seleccionar métodos apropiados para lograr una mejor clasificación de los productores agropecuarios.

Una adecuada clasificación de los sistemas productivos puede apoyar el diseño de políticas agropecuarias para una zona (Landín, 1990), facilitar la definición de políticas de transferencia tecnológica (Suárez y Escobar, 1990; Álvarez y Paz, 1998) y ayudar al conocimiento de la dinámica de desarrollo de una región o al diseño y gestión de proyectos de desarrollo (Berdegué et al., 1990).

Pero los sistemas productivos no están formados por explotaciones homogéneas; existe una gran diversidad de éstas, con diferentes caracteres físicos, socioeconómicos o técnicos. El conocimiento en profundidad de un sistema agrario debe partir de una descripción de los tipos de explotación existentes. Tradicionalmente se ha superado este problema al definirlos con un criterio único de agrupación (pequeñas, medianas y grandes, por ejemplo).

Con el objetivo de vencer esta dificultad, es necesario clasificar y tipificar las explotaciones agropecuarias con el supuesto básico de que la realidad de éstas se explica por medio de innumerables variables que actúan conjuntamente, y no por su forma unitaria (Radrizzani, 2000). En este sentido, las técnicas de análisis multivariado son herramientas idóneas para la clasificación de fincas.

En el presente trabajo se ha seleccionado como caso de aplicación la zona de regadío del río Dulce de Santiago del Estero. La tipificación multidimensional ha sido escasamente aplicada en el sector agropecuario de esta provincia. Los trabajos de Álvarez y Paz (1997), Radrizzani (2000), Montenegro (2000) y Paz (2002) constituyen algunas de las pocas incursiones en el tema.

Esta región es una típica provincia mediterránea (Mapa 1), con dos importantes ríos que la atraviesan en dirección noroeste-sudeste y que delimitan las zonas más densamente pobladas del territorio, a partir del uso agrícola de sus aguas (Mapa 2).

La estructura económica de la provincia tuvo como principal soporte productivo las actividades de origen agrícola (algodón, soja, maíz, cebolla, alfalfa y sorgo) y la producción ganadera de bovinos y caprinos. En particular, los primeros proceden básicamente de

\section{DeSarrortrollo}




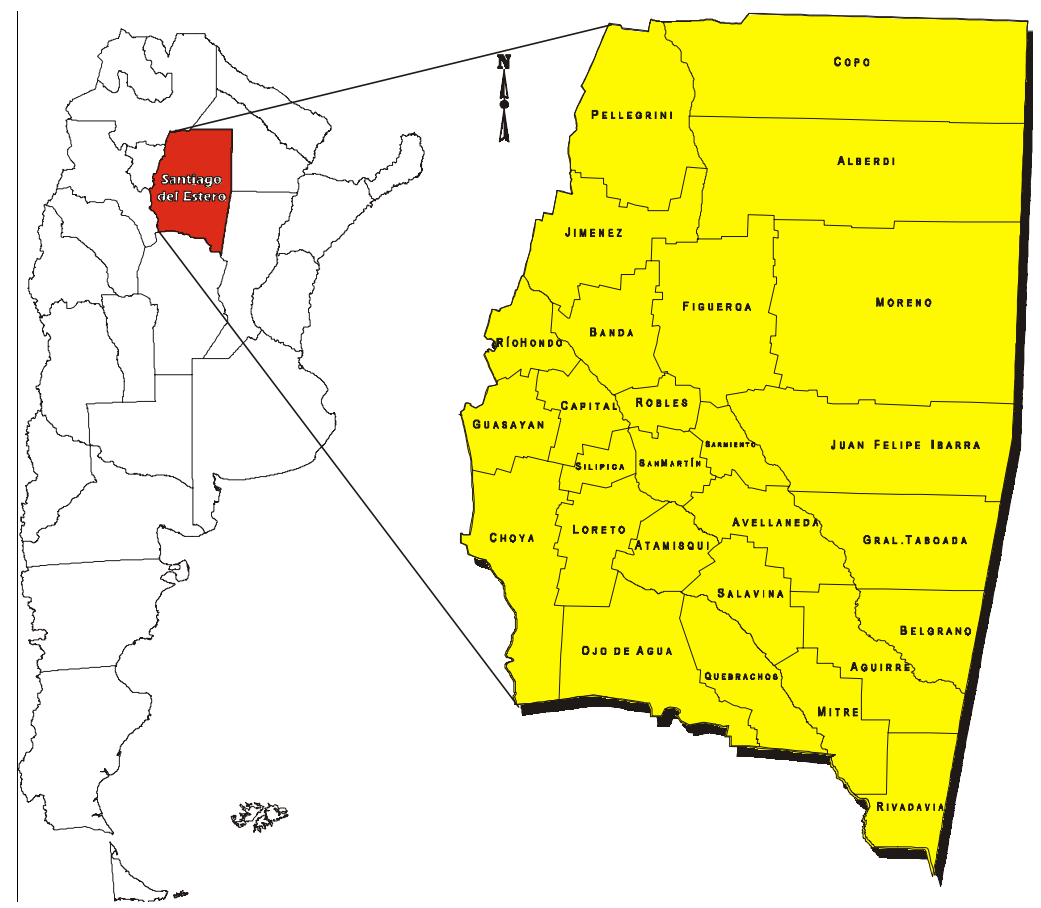

Mapa 1. Ubicación de Santiago del Estero en el contexto nacional.

Fuente: Laboratorio de Percepción Remota, Facultad de Ciencias Forestales, unse, 2002.

la región central de la provincia delimitada por sus dos grandes ríos, en especial el río Dulce y, secundariamente, el río Salado, los cuales la transforman en la zona más rica y productiva de la geografía provincial. Esta zona es denominada área de riego del río Dulce de Santiago del Estero.

Hasta mediados de la década de los años setenta, el sector agropecuario constituía la actividad más relevante de la economía provincial y generaba alrededor de 30\% del producto interno bruto (PIB), en gran parte por la expansión experimentada por su tradicional producción agrícola de regadío. A partir de ese periodo, comenzó a registrarse un continuo descenso. El sector rural llegó a disminuir su participación a 21\% en 1985 y a menos de 16\% en 1997, con una clara tendencia hacia el estancamiento (CFI-DGEYC, 1998).

Después del explosivo crecimiento de la producción de regadío en los años sesenta y setenta, se verificó una sostenida y profunda contracción de la superficie cultivada, situación que ha llegado hasta el presente (Jañez y Antuña, 2001). Las causas deben buscarse, entre otros aspectos, en el modelo de descentralización del Estado, la falta de políticas de apoyo al sector, los problemas de salinización de los suelos, la insuficiencia del sistema de drenaje y el abandono de las tareas de mantenimiento del sistema de riego.

Este escenario provocó el deterioro de las condiciones de vida de los campesinos y el empobrecimiento, el abandono de la producción y la concentración de la tierra; no obstan- 
Mapa 2. Hidrografía y relieve de Santiago del Estero.

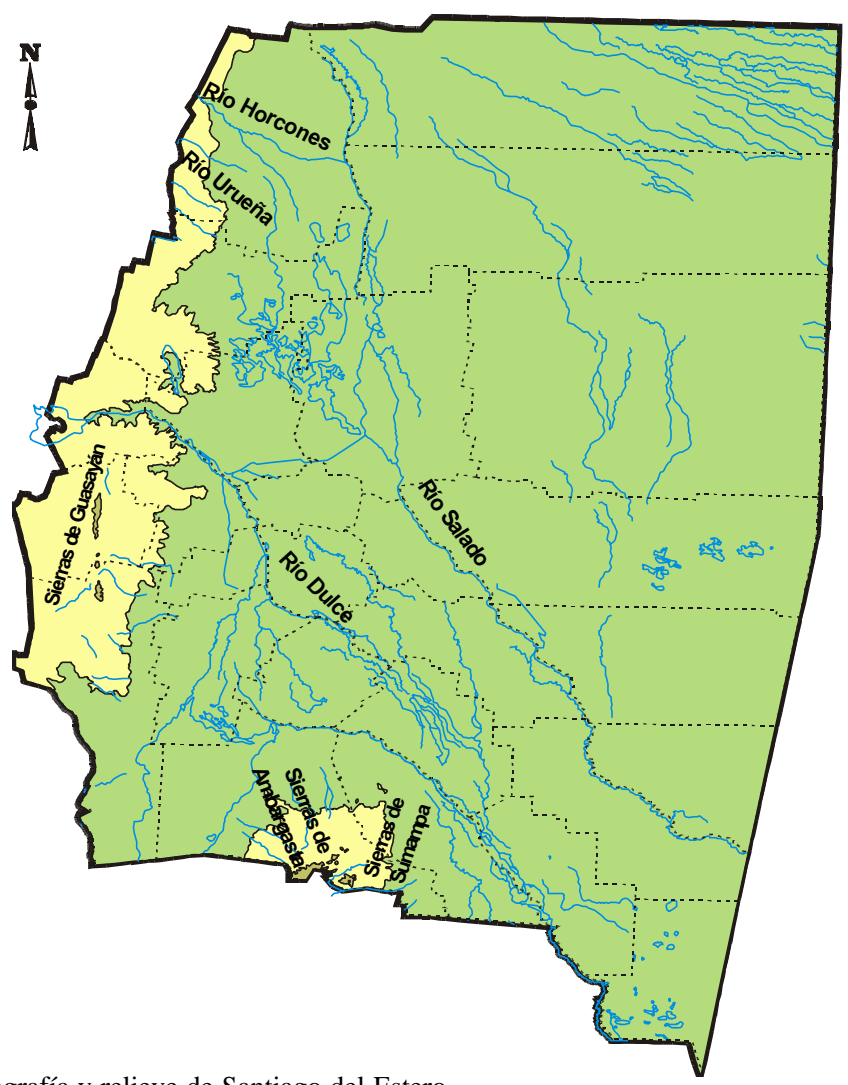

Fuente: Laboratorio de Percepción Remota, Facultad de Ciencias Forestales, UnSE, 2002.

te, las necesidades de ajuste estimularon respuestas de adaptación de los segmentos más capitalizados. En particular, los productores medianos y grandes, integrados al mercado, presentan mayor capacidad de adecuación mediante respuestas tecnológicas y económicas (Jañez et al., 1990). Por el contrario, los sectores minifundistas requieren un gran esfuerzo de asistencia estatal.

Por tanto, la hipótesis general de este trabajo es que el desarrollo de la zona estudiada pasa por el fortalecimiento de los sectores de rasgos más decididamente eficientes, en especial las explotaciones de tipo empresarial.

La escasa información local acerca de los distintos actores sociales y la inexistencia de estudios integrales, con una visión amplia y global de los problemas, limita el conocimiento de la realidad y la posibilidad de llevar a cabo acciones para mejorarla. Sin embargo, cualquier propuesta para superar esta crisis debe partir de una tarea de diagnóstico y, en ésta, no puede estar ausente el reconocimiento de los diferentes tipos de sistemas de producción con sus conflictos, limitaciones y potencialidades. La tipificación de los sistemas productivos constituye, en este sentido, una herramienta metodológica capaz de cap- 
tar esta diversidad; responde al criterio general de que cada tipo de explotación agropecuaria constituye una modalidad específica de organización de los recursos productivos y expresa una lógica particular de funcionamiento (Escobar y Berdegué, 1990). Todos estos aspectos deben ser considerados para elaborar propuestas de políticas que promuevan una gestión eficiente de la agricultura de regadío, condición necesaria para el desarrollo regional del área.

\section{Objetivos del trabajo}

Bajo la premisa de fortalecer el segmento de productores con mayor eficiencia productiva, adecuación y respuesta al mercado, el objetivo general de este trabajo es clasificar y tipificar una muestra de explotaciones rurales del estrato correspondiente a medianos y grandes productores, que pueda ser utilizada para la generación y transferencia de alternativas tecnológicas coherentes con las circunstancias de la agricultura de riego de Santiago del Estero.

El trabajo de tipificación de las explotaciones agrarias tiene dos propósitos posteriores que no son abordados en el marco del presente trabajo: $a$ ) conformar una imagen ordenada de la agricultura de irrigación, hecho que significa un aporte a la elaboración de un diagnóstico provincial de los sistemas productivos agropecuarios, y b) diseñar modelos de optimación productiva, en los cuales se proponga un plan de producción en función de los recursos disponibles (Coronel de Renolfi y Ortuño, 2004).

\section{El área de estudio}

El escenario de esta investigación es la zona rural de regadío ubicada en la provincia de Santiago del Estero, Argentina, perteneciente a la región fitogeográfica del Chaco (Ledesma, 1992). Es el área de producción agropecuaria con mayor concentración en la provincia; genera $60 \%$ del producto bruto agropecuario provincial por la incidencia de los cultivos de riego (alfalfa, algodón, hortalizas, maíz y soja) y, en menor medida, por las actividades pecuarias (UESRRD, 1992). Además, es la única zona de la provincia que dispone de irigación asegurada.

El sistema de regadío del río Dulce ocupa una superficie de 300000 ha; está dotado de una vasta red de canales con $1100 \mathrm{~km}$ de recorrido (Mapa 3). Potencialmente, es posible el riego de 120000 ha (UESRRD, 1992). Mientras en 1970 el área cultivada era de 90000 ha, en 1980 descendió a sólo 30000 ha. Los datos más recientes indican aproximadamente 73000 ha en actividad (Radrizzani, 2000).

El área de irrigación combina, en forma dominante, la economía campesina de autoconsumo con la empresarial, en un sistema de gran heterogeneidad social. Los pequeños productores, con unidades menores a 20 ha, representan $75 \%$ de los agricultores de la zona y

\section{DeSarrollo}




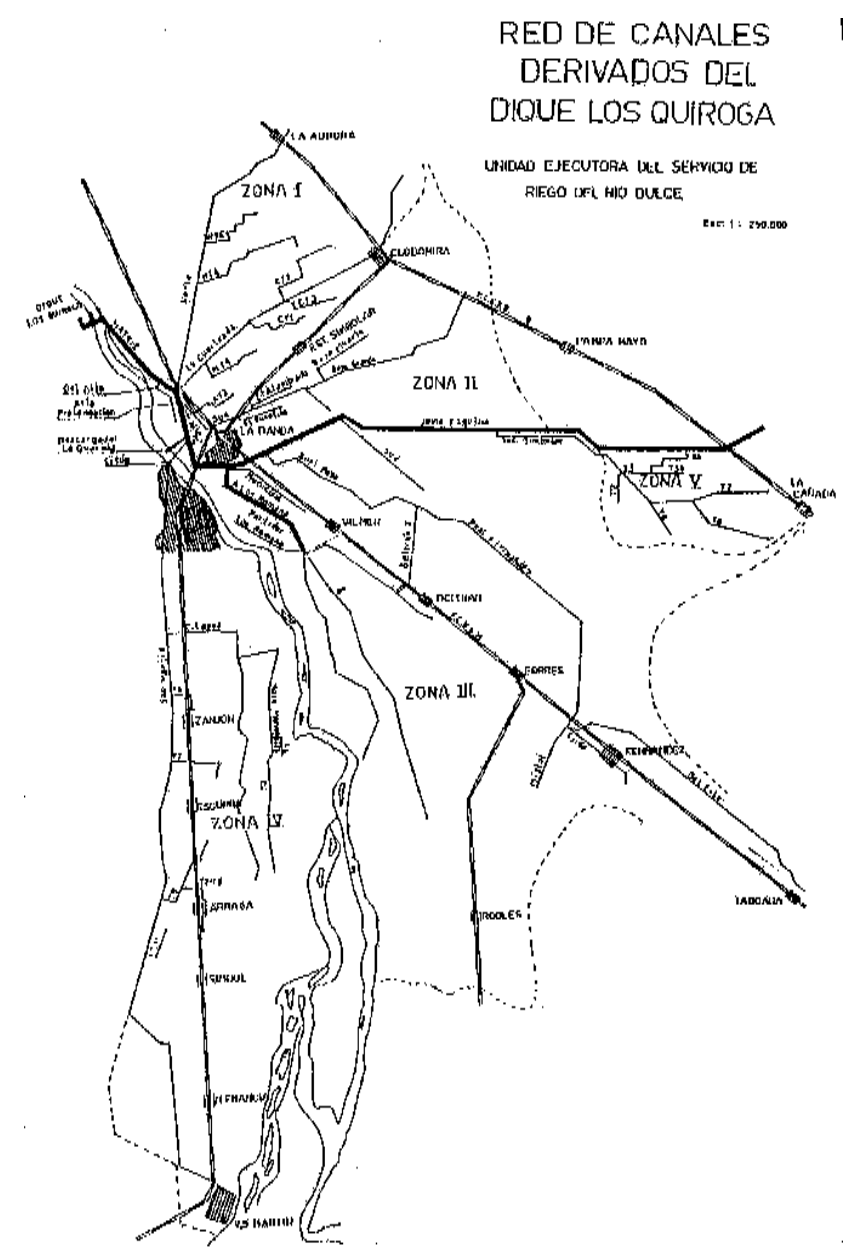

Mapa 3. Plano del sistema de riego del río Dulce, red de canales.

Fuente: Unidad Ejecutora del Servicio de Riego del Río Dulce (UESRRD), 1992.

explotan $20 \%$ de la superficie total; los productores medianos y grandes, con más de 20 ha, representan una cuarta parte del total, pero posen $80 \%$ del área (Radrizzani, 2000).

\section{Instrumentos de recolección de información}

La recopilación de los datos de campo se hizo mediante la realización de una encuesta de tipo estructurada, efectuada en una muestra de 152 explotaciones agropecuarias pertenecientes al área de riego del río Dulce de Santiago del Estero, entre los meses de diciembre de 2001 y mayo de 2002.

Estructuralmente, la encuesta contiene las siguientes partes: $a$ ) identificación de la explotación agropecuaria; $b$ ) estrategias agroproductivas; $c$ ) actividad pecuaria; $d$ ) actividad

\section{DeSarrollo}


forestal; $e$ ) aspectos ecológicos; $f$ ) aspectos sociales y $g$ ) forma social del trabajo. A partir de esta estructura, se confeccionó el cuestionario, que incluye preguntas de carácter social, productivo, ecológico y económico. Se obtuvieron 52 variables primarias, cualitativas y cuantitativas, que fueron definidas y codificadas para su posterior procesamiento y análisis.

\section{Unidad de análisis}

La conveniencia de obtener información primaria a partir de una muestra exigió definirla a priori. Los requisitos de pertenencia de la población fueron los siguientes:

1. Que sea una explotación rural, mediana o grande, de propiedad privada.

2. Que pertenezca a la zona de riego del río Dulce.

3. Que tenga una superficie igual o superior a 20 ha.

4. Que en ella se desarrolle actividad agropecuaria y/o forestal bajo el control del establecimiento. ${ }^{1}$

5. Que produzca bienes destinados a su comercialización en el mercado.

El trabajo de preparación en laboratorio, previo a las salidas a campo, consistió en ubicar en una imagen de satélite las explotaciones sorteadas y verificar si pertenecían a la muestra. Los requisitos 2 y 3 fueron examinados en laboratorio de manera inmediata, sin necesidad de verificar en el terreno. ${ }^{2}$ Por el contrario, las condiciones 1,4 y 5 solamente fueron verificadas in situ. El límite mínimo de tamaño se definió a partir de la consulta de fuentes secundarias y otros estudios en la zona (Jañez et al., 1990; Radrizzani, 2000).

La unidad objeto de análisis fue, por lo tanto, la propiedad rural, identificada aquí como una explotación productiva agropecuaria (EPA) y definida como una unidad privada de organización de la producción, mediana o grande, con una superficie igual o mayor a 20 ha en plena actividad, ubicada dentro de los límites de la zona de riego del río Dulce que produce bienes agrícolas, pecuarios o forestales — en forma exclusiva o combinada, destinados al mercado-, que tiene un productor que asume la gestión, dirección y los riesgos de la actividad y que utiliza, en todas las parcelas que la integran, los mismos medios de producción.

Sobre esta base, el estudio se circunscribió a los sistemas de producción rural —vinculados con el mercado agropecuario- que proporcionan retornos mediante un proceso de producción primaria; se excluyeron aquellas explotaciones que generan bienes para autoconsumo y no destinan excedentes al mercado.

1 En el momento de seleccionar la muestra, no había modo de verificar si la explotación estaba en actividad efectiva, salvo la información que refleja la imagen de satélite (del año 2000); pero una vez en el terreno, se pudo constatar que algunos predios sorteados están actualmente abandonados.

2 El software utilizado permite medir la superficie de cada lote del catastro. 


\section{Estrategia de muestreo}

La encuesta se realizó sobre una muestra aleatoria de productores rurales, utilizando el método de muestreo por conglomerado en dos etapas (Scheaffer et al., 1987) también denominado muestreo por conglomerado bietápico (González García et al., 1993). Éste proporciona más información por unidad de coste que cualquiera de los otros diseños estadísticos (Cochran, 1990; Scheaffer et al., 1987).

El diseño de muestreo por conglomerado se aplica en poblaciones muy extensas y parceladas, donde la localización de la muestra seleccionada supone grandes desplazamientos y se espera mucha variabilidad de la población (González García et al., 1993). En el caso de este trabajo, son tres las razones fundamentales que llevaron a optar por este diseño: $a$ ) es efectivo para obtener una cantidad específica de información al mínimo coste, cuando no se encuentra disponible un buen marco que filtre los elementos de la población; $b$ ) es menos costoso que el muestreo aleatorio simple y que el estratificado, cuando el coste por obtener observaciones se incrementa con la distancia que separa los elementos por estudiar, y c) los elementos dentro del conglomerado están geográficamente cerca unos de otros y, por lo tanto, se reducen gastos de transporte, tiempo de traslado y tiempo de toma de datos.

Como cada aglomeración contenía demasiadas unidades de análisis, se seleccionó primero una muestra aleatoria de conglomerados y, posteriormente, se tomó una muestra aleatoria de explotaciones dentro de cada uno de éstos, de manera que resultó un muestreo en dos etapas.

A fin de facilitar la ubicación de los conglomerados y de las explotaciones por entrevistar en cada uno de éstos, se empleó una malla cuadriculada superpuesta sobre la imagen de satélite y el plano catastral de la zona de estudio.

\section{La tipificación de sistemas productivos}

Las explotaciones agropecuarias se analizaron como sistemas productivos en los cuales actúan, simultáneamente, innumerables variables estructurales, sociales, económicas y ecológicas. Se intenta descubrir las relaciones que existen entre conjuntos de variables e individuos o unidades de estudio para los cuales se han medido dichas variables. Desde este enfoque, el objetivo de clasificar sistemas de producción y tipificar grupos homogéneos de productores rurales debe resolverse con una metodología que sea capaz de recoger la diversidad de la estructura y el funcionamiento de las unidades productivas (Escobar y Berdegué, 1990). La base metodológica del trabajo de tipificación es el análisis estadístico multivariante. La utilidad de los métodos multivariantes consiste en que permiten construir clasificaciones de conjuntos, considerando simultáneamente diversas variables. 


\section{Análisis estadístico multivariante}

El análisis estadístico multivariante ordena, resume y clasifica datos provenientes de poblaciones en las cuales se han medido dos o más características.

Del conjunto de técnicas diversas que ofrece el análisis multivariante, se utilizaron las siguientes: $a$ ) análisis de componentes principales (ACP), $b$ ) factorial, y c) cluster. El objetivo de las dos primeras es la simplificación estructural, y el de la última, la clasificación o agrupación (Manly, 1986; De Olivera et al., 1990; Afifi y Clark, 1999).

El ACP consiste en la transformación de variables originales en nuevas no correlacionadas. Establece las relaciones entre grupos de variables que mantienen una varianza interna definida por un mismo fenómeno y dependiente de razones de similar poder explicativo. Esta técnica fue aplicada a fin de simplificar la descripción de las variables que se midieron en cada finca, ganar mayor entendimiento de sus interrelaciones y, de este modo, reducir la dimensión del problema sin perder mucha información original (escogiendo un número de componentes que expliquen la mayor varianza total que fuera posible).

Afifi y Clark (1999) y Montenegro (2000) advierten que, en la práctica, la explicación de los componentes principales no es sencilla; a menudo no se logra en la extracción inicial de éstos y la aplicación del análisis factorial puede mejorar la interpretación de los resultados en el marco del problema que se examina. Por esta razón, para facilitar la comprensión de las relaciones entre variables, se empleó posteriormente el análisis factorial, con el cual se logró aclarar un alto porcentaje de la información original con muy pocos factores. Esta herramienta permite obtener nuevas variables sintéticas distintas. La técnica consiste en representar cada una de las variables en estudio como una combinación lineal de un conjunto menor de factores.

Dado que cada factor principal es una variable sintética construida a partir de las variables originales, cada observación (de las fincas) pudo ser identificada por sus coordenadas respecto de cada uno de los factores. Éstos fueron utilizados como variables de clasificación en la técnica de grupo o análisis cluster. Al respecto, Afifi y Clark (1999) señalan que puede resultar apropiado aplicar alguna de estas dos técnicas antes de realizar el análisis cluster, por la ventaja de reducir el número de variables originales. La aplicación esta técnica permitió formar grupos homogéneos de explotaciones, en los cuales la variabilidad dentro del grupo es mínima y, entre los grupos, es máxima.

\section{Revisión y selección de variables}

De las 52 variables originales, se seleccionaron aquellas que efectivamente puedan contribuir al análisis multivariante de tipificación. Para ello, se calculó el coeficiente de variación (Cv) de las variables, descartándose aquéllas con uno inferior a 50\% (Escobar y Berdegué, 1990; Radrizzani, 2000; Montenegro, 2000) y, por lo tanto, con poco poder discriminante. 
A continuación se calculó una matriz completa de correlaciones entre las variables retenidas, con el objetivo de identificar grupos de variables fuertemente vinculadas entre sí y que, por consiguiente, puedan conducir a que un único fenómeno (por ejemplo, el tamaño de la explotación) estuviese representado múltiples veces en los análisis posteriores. De esta forma se identificaron diversas variables con alto porcentaje de información superflua, es decir, altamente correlacionadas desde el punto de vista de la tipificación. Sobre este aspecto, varios estudios destacan la importancia de determinar variables representativas, seleccionarlas de acuerdo con su consistencia interna con otras del conjunto y eliminar aquellas muy vinculadas o las que no guardan ninguna relación con las demás (Landín, 1990; Hart, 1990; Berdegué et al., 1990; Paz, 2002).

En el proceso de selección se llegó a una lista de once variables: SUP, AÑOS, POT, ING, ACTIV, ESTUDIO, MEC, FLIAR, TEMP, JUB y VIVE. ${ }^{3}$ Éstas satisfacen los requisitos de contribuir a la tipificación por su escasa correlación entre sí y de ser expresiones relevantes de los aspectos estructurales, socioeconómicos y de gestión productiva de las explotaciones.

\section{Resultados y discusión}

Los resultados obtenidos del agrupamiento permitieron caracterizar los sistemas tipo de producción que condujeron a la clasificación de cuatro explotaciones representativas del área de riego del río Dulce.

\section{Resultados del trabajo de campo}

$\mathrm{Al}$ descartar seis parcelas propiedad del Estado, la muestra quedó integrada por 146 explotaciones agropecuarias, de las cuales 75 resultaron improductivas y 71 en actividad (ocho explotaciones pertenecientes a productores que no quisieron participar en la investigación). De las 63 encuestas efectuadas, fueron eliminadas seis porque se tuvieron dudas acerca de la calidad y veracidad de la información entregada por el productor, de manera que se lograron obtener 57 observaciones válidas. Los resultados del trabajo de campo, expresados en porcentajes, se reflejan en la Gráfica 1.

Con respecto al nivel de inactividad productiva, es importante resaltar el porcentaje de abandono de tierras en la zona, el cual representa 52\% del total de la muestra. Radrizzani (2000) señala un porcentaje de parcelas improductivas de $45 \%$.

SUP: superficie total en actividad. AÑos: antigüedad en la explotación. POT: potencia de tracción por unidad de superficie. ING: ingreso agrario por unidad de superficie. ACTIV: actividad principal. ESTUDIO: nivel de instrucción. MEC: grado de mecanización. FLIAR: empleo de mano de obra familiar. TEMP: empleo de mano de obra transitoria. JUB: planes de jubilación. VIVE: residencia habitual del productor.

\section{DeSarrortollo}




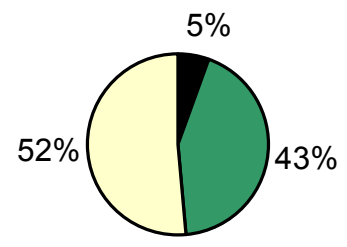

Sin respuesta $\square$ Con respuesta $\square$ Inactivas

Gráfico 1. Resultados de la toma de datos de campo.

\section{Resultados del análisis de componentes principales}

Mediante este procedimiento se extrajeron cuatro componentes principales que, en total, dan cuenta de $64.08 \%$ de la varianza de la matriz de los 57 casos por las 11 variables seleccionadas, según se indica en el Cuadro 1.

Cuadro 1

Primer ciclo del ACP con once variables

\begin{tabular}{ccccc}
\hline \multicolumn{5}{c}{ Extraction: principal components (matriz de 57 x 11) } \\
\hline \multirow{2}{*}{ Componentes } & $\begin{array}{l}\text { Varianza del } \\
\text { componente }\end{array}$ & \% varianza total & $\begin{array}{c}\text { Varianza } \\
\text { acumulada }\end{array}$ & $\begin{array}{c}\text { \% de varianza } \\
\text { acumulada }\end{array}$ \\
\hline 1 & 2.442900 & 22.20818 & 2.442900 & 22.20818 \\
2 & 2.170319 & 19.73017 & 4.613219 & 41.93835 \\
3 & 1.344143 & 12.21948 & 5.957362 & 54.15784 \\
4 & 1.092057 & 9.92779 & 7.049419 & 64.08563 \\
\hline
\end{tabular}

Sin embargo, para simplificar la interpretación analítica y gráfica del problema, el propósito era obtener, como máximo, dos o tres factores que explicaran la mayor cantidad posible de la varianza total; idealmente, el punto de corte es entre 70-80\% (Manly, 1986; Afifi y Clark, 1999). En este caso, el primer ciclo del ACP no dio resultados satisfactorios: los tres primeros componentes sólo representa $54.15 \%$ de la varianza acumulada. En consecuencia, se reinició el ciclo añadiendo un filtro de apreciación técnica en la mayor o menor relevancia de la información contenida en cada variable y se impuso la condición de que éstas debían ayudar a expresar la conformación de la estructura, la gestión y la realidad socioeconómica de las explotaciones. De esta forma, del grupo de once variables filtradas fueron eliminadas cuatro, para llegar a una lista definitiva de siete descriptores de las explotaciones agropecuarias: SUP, ACTIV, ESTUDIO, MEC, FLIAR, TEMP y JUB.

Las variables РОт у MEC expresan atributos similares, desde la óptica de la estructura predial. Se optó por conservar la segunda, previa prueba comparada de los resultados con una y otra. La variable ING, aparentemente importante, fue eliminada en razón de que repre- 
senta valores de una realidad circunstancial, de una situación de corte temporal. Que una variable sea considerada relevante desde el punto de vista teórico no garantiza que tenga una varianza razonable dentro de la muestra. Para la variable vive, también eliminada, la experiencia de campo delató una relación estrecha con sup: una finca pequeña se corresponde con un productor residente en la explotación.

En el segundo ciclo del ACP los resultados mejoraron significativamente, ya que los primeros tres componentes explican 68\% de la variabilidad total (Cuadro 2).

Cuadro 2

Segundo ciclo del ACP con siete variables

\begin{tabular}{ccccc}
\hline \multicolumn{5}{c}{ Extraction: principal components (matriz de 57 x 7) } \\
\hline Componentes & $\begin{array}{l}\text { Varianza del } \\
\text { componente }\end{array}$ & \% varianza total & $\begin{array}{c}\text { Varianza } \\
\text { acumulada }\end{array}$ & $\begin{array}{c}\text { \% de varianza } \\
\text { acumulada }\end{array}$ \\
\hline 1 & 1.921309 & 27.44727 & 1.921309 & 27.44727 \\
2 & 1.770245 & 25.28921 & 3.691554 & 52.73648 \\
3 & 1.069432 & 15.27760 & 4.760986 & 68.01408 \\
\hline
\end{tabular}

Cada componente principal tiene varianza máxima no correlacionada con los restantes. El primero es la combinación de variables que expresa la mayor varianza del fenómeno, con valor de 1.92 .

Una vez seleccionados los componentes, se examinaron los coeficientes con el fin de asignar una interpretación a cada uno de ellos. Los componentes principales se interpretan en el contexto de las variables con coeficientes altos, en valor absoluto (Manly, 1986). Para interpretar los primeros se calculó la carga factorial (factor loading) o peso que las variables tiene en cada componente (Cuadro 3).

Cuadro 3

Cargas factoriales de las variables en los componentes principales

\begin{tabular}{|c|c|c|c|}
\hline \multicolumn{4}{|c|}{$\begin{array}{c}\text { Extracción de los componentes principales (no rotados) } \\
\text { (Marca en cargas factoriales }>.600000)\end{array}$} \\
\hline \multicolumn{4}{|c|}{ Cargas factoriales } \\
\hline Variables & Componente 1 & Componente 2 & Componente 3 \\
\hline SUP & -.352991 & .366129 & -.696061 \\
\hline ACTIV & .298021 & .760594 & .270878 \\
\hline ESTUdiO & -.398711 & .689748 & .322638 \\
\hline MEC & -.791356 & -.169865 & -.146682 \\
\hline FLIAR & .719256 & .121105 & .030508 \\
\hline TEMP & -.546689 & -.315129 & .620409 \\
\hline JUB & -.326306 & .662655 & -.010295 \\
\hline Varianza explicada & 1.921309 & 1.770245 & 1.069432 \\
\hline Proporción del total & .274473 & .252892 & .152776 \\
\hline
\end{tabular}


Del este cuadro se infiere que el primer componente tiene alto grado de dependencia en las variables MEC y FLIAR; el segundo, sobre las variables ACTIV, ESTUDIO y JUB, y el tercero, sobre las variables SUP y TEMP. No obstante, estos resultados no permitieron adjudicar una explicación satisfactoria a los tres componentes.

\section{Resultados del análisis factorial}

Ante los resultados poco satisfactorios para interpretar los componentes principales, se procedió a aplicar el análisis factorial para hallar nuevos factores cuyas cargas fueran fáciles de estudiar, con el fin de asignarles sentido y nombre a cada uno de ellos. Se utilizó el método de rotar los factores provisorios iniciales, probando varias técnicas de rotación ortogonal. Esta rotación mantiene la calidad global de la representación y la capacidad conjunta de los factores para retener la información de cada variable, sin modificar el porcentaje de varianza acumulada. Finalmente, se optó por la rotación varimax, que otorgó el mejor significado a los factores.

Los resultados de este procedimiento definieron tres factores que explican $68 \%$ de la varianza de las siete variables y que agrupan a las mismas en forma más específica. El Cuadro 4 permite observar las cargas factoriales de cada una de las variables sobre los tres factores. En el mismo, se observa que la varianza explicada por cada factor ha cambiado ligeramente con respecto a la de cada componente de la extracción inicial, pero la proporción de varianza total se mantiene en $68 \%$, porcentaje de explicación aceptable.

Después, se procedió a asignar una interpretación física de los factores extraídos, en el contexto de la caracterización de las unidades agropecuarias de la zona de riego.

El factor F1 tiene correlación elevada con las variables MEC (grado de mecanización), TEMP (empleo de mano de obra transitoria) y FLIAR (empleo de mano de obra familiar).

Cuadro 4

Cargas factoriales de las variables en los factores rotados

\begin{tabular}{|c|c|c|c|}
\hline \multicolumn{4}{|c|}{$\begin{array}{c}\text { Extracción de los componentes principales (rotación varimax) } \\
\text { (marca en cargas factoriales }>.600000)\end{array}$} \\
\hline \multicolumn{4}{|c|}{ Cargas factoriales } \\
\hline Variables & Factor 1 & Factor 2 & Factor 3 \\
\hline SUP & .189467 & .140267 & .829206 \\
\hline ACTIV & -.500109 & .700055 & -.022566 \\
\hline ESTUDIO & .187051 & .838735 & .018831 \\
\hline MEC & .797052 & -.002246 & .203265 \\
\hline FLIAR & -.719609 & -.067803 & -.102428 \\
\hline TEMP & .647419 & .108325 & -.593463 \\
\hline JUB & .109329 & .664925 & .302681 \\
\hline Varianza explicada & 1.905228 & 1.671690 & 1.184068 \\
\hline Proporción del total & .272175 & .238813 & .169153 \\
\hline
\end{tabular}

\section{DeSarrollo}


Por lo tanto, al primer factor - el cual puede ser visto como una medida del uso de capital y mano de obra en las explotaciones rurales- se le llamó recursos del predio. Es importante señalar que la nueva variable expresa $27.21 \%$ de la varianza de las siete variables seleccionadas, lo cual indica que F1 es el factor más influyente en el análisis y, consecuentemente, el que mejor explicará las diferencias entre los distintos sistemas productivos.

Por su parte, el factor F2 posee una correlación alta con las variables ESTUdio (nivel de instrucción), ACTIV (actividad principal) y JUB (planes de jubilación). Dado que este factor puede interpretarse como indicativo de la caracterización social y la orientación del productor, el resultado de F2 fue denominado realidad socio-productiva. Ésta explica $23.88 \%$ de la varianza de las siete variables.

De forma similar se interpretó el factor F3. Presenta correlación elevada con una sola variable, sup (superficie total en actividad) y mide el tamaño o escala de producción. La nueva variable se denominó dimensión y expresa $16.91 \%$ del total de la varianza de las variables. En el Cuadro 5 se resume la carga factorial de las variables en los factores rotados, el porcentaje de la varianza total que explica cada factor y la interpretación física asignada a los mismos.

\section{Resultados del análisis cluster}

Con la información resultante del análisis factorial, se realizó el análisis cluster con el propósito de formar grupos de sistemas productivos similares respecto de las tres nuevas variables creadas: recursos del predio, realidad socio-productiva y dimensión.

Existe una cierta contradicción entre el objetivo del presente trabajo y sus propósitos posteriores, en relación con el número máximo de tipologías para obtener del análisis cluster. Desde el punto de vista de la elaboración de un diagnóstico de los sistemas productivos de la zona de riego del río Dulce, es importante aceptar todos los que tengan un mínimo de relevancia. Sin embargo, desde el punto de vista que selecciona explotaciones

Cuadro 5

Rotación de factores y su denominación

\begin{tabular}{clccl}
\hline \multirow{2}{*}{ Factores } & Variables & Carga factorial & $\begin{array}{c}\text { Proporción de } \\
\text { varianza explicada }\end{array}$ & Interpretación \\
\hline \multirow{3}{*}{ Primero } & MEC & 0.797 & & \\
& FLIAR & 0.719 & 0.272 & Recursos del predio \\
& TEMP & 0.647 & & \\
\multirow{2}{*}{ Segundo } & ESTUDIO & 0.838 & & Realidad socio-productiva \\
& ACTIV & 0.700 & 0.238 & Dimensión \\
\hline \multirow{2}{*}{ Tercero } & JUB & 0.664 & & \\
\hline
\end{tabular}


representativas de cada grupo para el posterior diseño de modelos de optimación en las mismas, es evidente que el número de grupos no puede ser muy elevado. La decisión fue, por lo tanto, conformar no más de cuatro o cinco conjuntos de sistemas de producción.

Escobar y Berdegué (1990) aconsejan llevar a cabo un examen de validez y fiabilidad de la tipología resultante; sugieren repetir el ejercicio usando otras técnicas analíticas y comparar los resultados finales. El supuesto con el que opera esta validación es que las categorías deben ser semejantes, independientemente de los métodos utilizados para generarlas. Adoptando estas sugerencias en la aplicación del análisis cluster, se decidió ensayar con métodos jerárquicos y no jerárquicos a fin de contrastar sus resultados.

\section{Aplicación de un método jerárquico}

Se utilizó el algoritmo jerárquico ascendente empleando, como medida de distancia, la euclídea cuadrada. Fueron usadas las opciones del enlace completo y del método de Ward para comparar los resultados con dos métodos de amalgamiento, ${ }^{4}$ complementados con la representación gráfica de un diagrama de árbol o dendrograma. Cada una de las ramificaciones mostradas en éste tiene el potencial de ser seleccionada como grupo de fincas. El nivel al cual se decide hacer la selección de los conjuntos depende del balance que el investigador haga de los siguientes elementos: a mayor cantidad de tipos, mayores serán la homogeneidad intratipos, la heterogeneidad intertipos, el coste y el esfuerzo de la investigación posterior (Rodríguez Ocaña et al., 1998).

Los dendrogramas obtenidos para los dos métodos de amalgamiento se ilustran en las Gráficas 2 y 3 . En el dendrograma de la Gráfica 2 se observa que a una distancia de corte de diez se forman cuatro grupos. En la Gráfica 3, la formación de cuatro grupos se consigue cortando el dendrograma a una distancia de aproximadamente 40.

Los dos métodos de amalgamiento dieron resultados diferentes en cuanto al número de integrantes de los grupos. Montenegro (2000) señala que, en el método de amalgamiento de Ward, la varianza dentro de los conjuntos aumenta, por lo que éstos resultan más heterogéneos. Dicha afirmación llevó a evaluar los resultados del agrupamiento con algoritmos no jerárquicos.

\section{Aplicación de un método no jerárquico}

En este caso, se usó el algoritmo no jerárquico de K-medias que, a diferencia de los jerárquicos, requiere fijar a priori el número de grupos. Para su aplicación, se solicitó la formación de cuatro grupos ( $G 1, G 2, G 3$ y G4). Por defecto, el programa emplea la distancia euclídea como medida de distancia.

4 La regla de amalgamiento es el criterio empleado para unir o separar grupos; depende del modo en que se mida la distancia entre dos grupos (De Olivera et al., 1990).

\section{DeSarrollo}




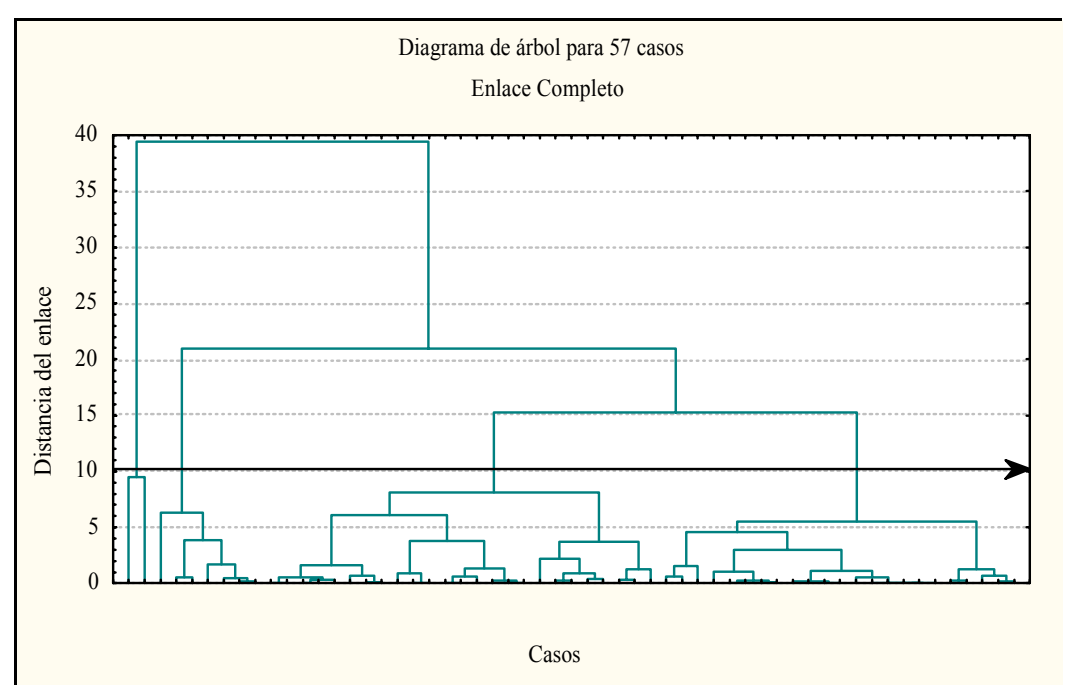

Gráfica 2. Dendrograma del análisis cluster con el método de enlace completo.

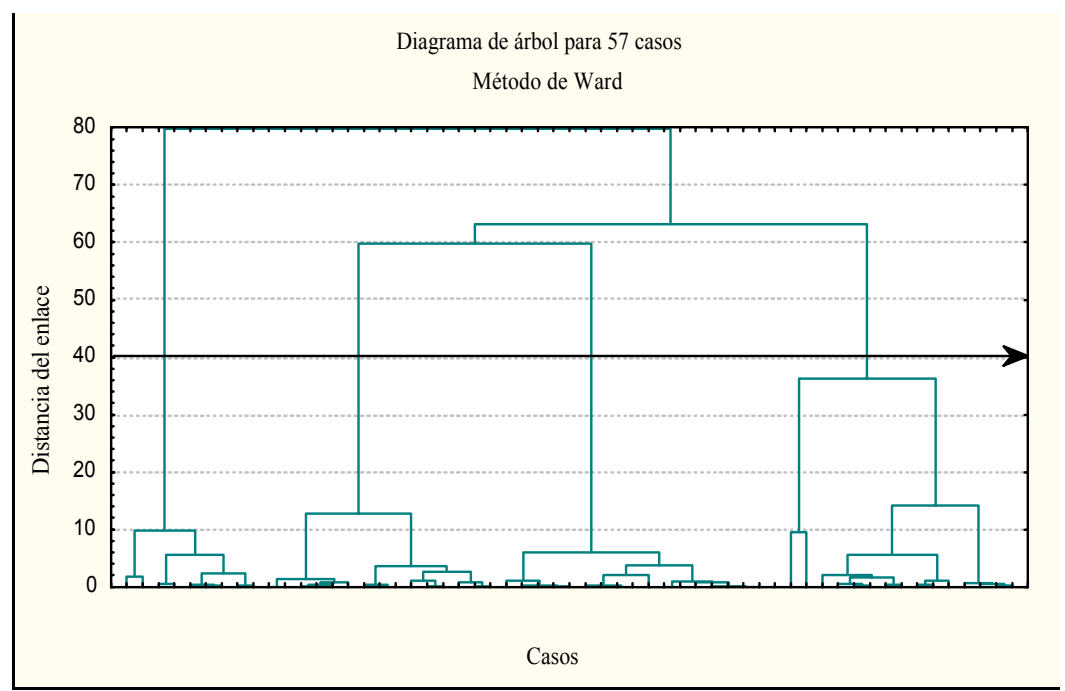

Gráfica 3. Dendrograma para el análisis cluster con el método de Ward. 


\section{Contraste entre los tres métodos de agrupamiento}

Para cotejar los resultados obtenidos en los tres métodos de agrupamiento, se examinaron las diferencias con respecto al número y a la nómina de los integrantes de cada grupo. De la comparación pareada entre los tres métodos de agrupamiento, el método de K-medias presentó el mayor número de coincidencias. Este procedimiento ha ganado una aceptación mayor en la literatura que los métodos jerárquicos (Manly, 1986; Afifi y Clark, 1999). Por medio de estos conceptos, se decidió adoptar el agrupamiento resultante del método de K-medias, tomando como base los resultados numéricos que ofrece la salida del programa informático utilizado, ${ }^{5}$ tales como el análisis de la varianza y las medidas estadísticas de posición, entre otros.

El análisis de la varianza cuantifica la importancia relativa de cada factor en la determinación de los grupos, por medio del estadístico F. Un valor alto de F es una indicación de que el correspondiente factor es muy útil para separar los clusters (De Olivera et al., 1990). Los valores del ratio F se usan sólo con propósitos comparativos, no como un test de hipótesis (Afifi y Clark, 1999).

En el análisis de la varianza que se presenta en el Cuadro 6, puede advertirse que los valores de F2 son altos, lo cual revela que los tres factores contribuyeron a la discriminación entre los cuatro grupos $G 1, G 2, G 3$ y G4. El valor más elevado de F es de 45.6 para F1, indicando que los grupos son más diferentes en función de ese factor (variable recursos del predio). A la inversa, el valor de F para F3 es el más bajo, por lo que este factor tiene un papel menos importante en la definición de los conjuntos. Estos resultados coinciden con los del análisis factorial, cuando se analizaron los valores de la varianza de los factores: el factor F1 presentó la mayor varianza explicada.

Cuadro 6

Análisis de la varianza

\begin{tabular}{cccccc}
\hline \multicolumn{5}{c}{ Análisis de varianza } \\
\hline Factores & Entre factores & $G L(*)$ & Dentro del factor & $G L(*)$ & Prueba de F \\
\hline Factor 1 & 40.37989 & 3 & 15.62011 & 53 & 45.67048 \\
Factor 2 & 35.23302 & 3 & 20.76698 & 53 & 29.97307 \\
Factor 3 & 30.90530 & 3 & 25.09470 & 53 & 21.75733 \\
\hline
\end{tabular}

Los grupos resultantes son descritos cuantitativamente en el Cuadro 7, por medio de las correspondientes medidas de posición de las siete variables descriptoras.

$5 \quad$ El programa usado corresponde al paquete informático Statistica. 
Cuadro 7

Medidas estadísticas de posición de los grupos formados

\begin{tabular}{lcccc}
\hline Variables & Grupo I & Grupo 2 & Grupo 3 & Grupo 4 \\
\hline N (casos) & 7 & 16 & 10 & 24 \\
SUP & 617.21 & 164.18 & 47.95 & 72.33 \\
ACTIV & 1 & 3 & 3 & 1 \\
ESTUDIO & 1 & 2 & 1 & 1 \\
MEC & 3 & 3 & 1 & 3 \\
FLIAR & 0 & 0 & 1 & 0 \\
TEMP & 0 & 1 & 0 & 1 \\
JUB & 1 & 1 & 0 & 0 \\
\hline
\end{tabular}

\section{Comparación entre los análisis factorial y cluster}

Para comprobar la validez de la aplicación de las dos técnicas multivariantes, se realizaron gráficas de coordenadas en las cuales los factores constituyen los ejes y las observaciones son puntos en el plano. Así, se logró complementar el análisis cluster en cuanto a la relación entre los tipos de sistemas productivos, y entre éstos y los factores. El resultado se vuelve más robusto si las conclusiones son coincidentes por ambos caminos (Berdegué et al., 1990; Landín, 1990; Radrizzani, 2000).

Se construyeron tres planos bidimensionales de factores (F1 con F2, F1 con F3 y F2 con F3), en los cuales se representaron los casos identificados por el número que los asocia con el grupo al cual pertenecen ( $G 1, G 2, G 3$ y $G 4)$. El análisis consistió en identificar visualmente los grupos que se comportan en forma similar con relación a los tres factores.

Las gráficas obtenidas $(4,5$ y 6 ) confirmaron la semejanza de comportamiento, ya que los productores representados se agruparon en los ejes de los factores de acuerdo con los cuatro conjuntos formados.

La Gráfica 4 explica los casos en función de los factores recursos del predio (F1) y realidad socio-productiva (F2). El grupo G3 está claramente separado de los demás. También lo están G2 y G4, pero en ellos se incorporan entremezclados los casos del G1.

En la Gráfica 5 se representan todas las observaciones de los factores recursos del predio (F1) y dimensión (F3), por lo cual pone de manifiesto que la formación de los grupos en función de los factores F1 y F3 resultó más notoria que la mostrada en la Gráfica 4. El G3 se posiciona en forma similar. Los casos que conforman los grupos 1, 2 y 4 se concentran de manera más diferenciada y satisfactoria. No obstante, se observa que algunos casos de los grupos 2 y 4 se entremezclan entre sí.

La representación espacial de la nube de puntos, en términos de la realidad socioproductiva y la dimensión, se presenta en la Gráfica 6. En este caso, la formación de los grupos es bastante clara, salvo un leve solapamiento entre los grupos 3 y 4 . 


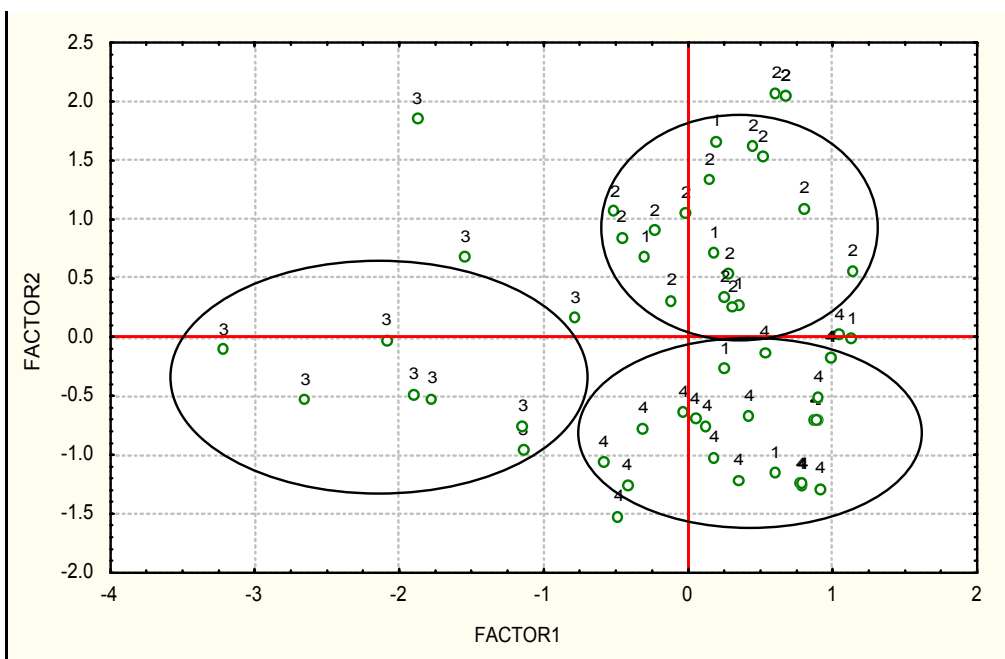

Gráfica 4. Agrupamiento de los casos según F1 y F2.

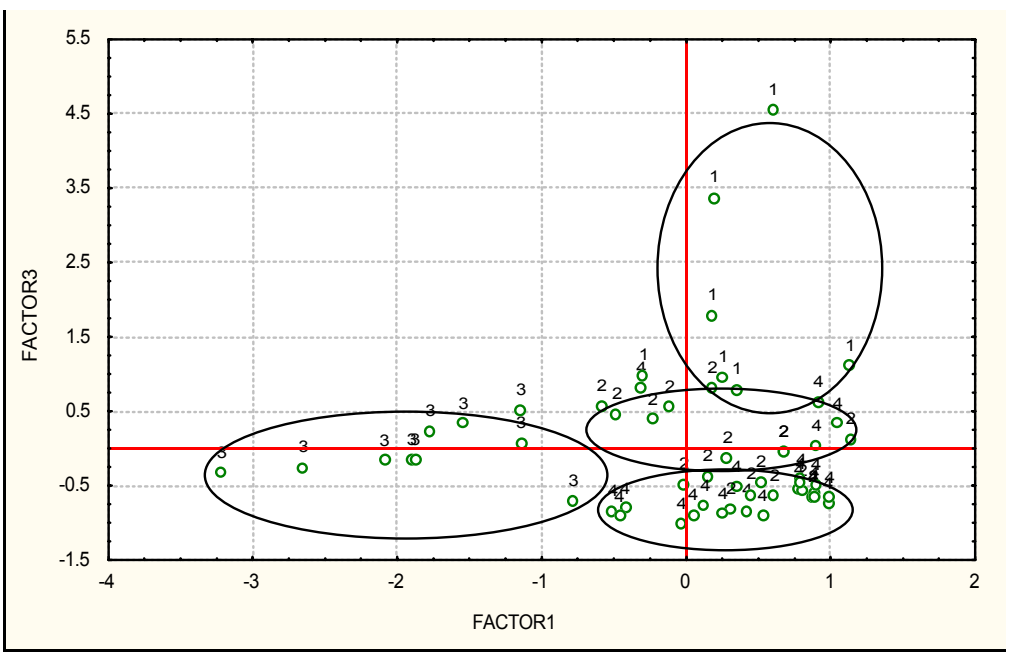

Gráfica 5. Agrupamiento de los casos según F1 y F3.

\section{Descripción de los grupos resultantes}

Una vez obtenida y verificada la tipificación de los grupos, se procedió a describir los tipos de sistemas de producción presentes en la zona de estudio, haciendo uso de las siete variables clasificatorias — según la información del Cuadro 7- y de muchas de las originales. Los cuatro grupos, bien diferenciados entre sí, se describen sintéticamente a continuación:

\section{Desparrofollo}


Grupo 1. Incluye $12.5 \%$ de la muestra y lo componen productores que reúnen las siguientes características:

- La superficie media en actividad es de 617 ha. Se detectan dos subgrupos bien diferenciados: el primero, con grandes extensiones de tierra trabajada superiores a 500 ha y que llegan hasta las 1700 ha, y el segundo, con escasa extensión de superficie activa que no supera las 40 ha.

- El nivel de instrucción es moderado bajo (más de la mitad sólo alcanzó a completar los estudios primarios); no llevan muchos años en la actividad rural (el promedio es de 17 años).

- Poseen un grado alto de mecanización y la potencia media de tracción, de 226 hp, es la más alta de todos los grupos.

- Poseen en nómina a empleados fijos; contratan personal transitorio en las épocas críticas de trabajo. No usan mano de obra familiar.

- En 57\% de los casos, la actividad principal es la agricultura exclusiva, con escaso grado de diversificación productiva. Producen maíz, soja, trigo y alfalfa para fardos; algunos cultivan cebolla, anquito y melón. Poseen suelos de buena calidad y no obtienen ingresos extraagrarios.

Este reducido grupo está formado por productores agrícolas tecnificados, con rasgos netamente empresariales, que hacen uso intensivo de sus recursos productivos, generan empleo, movilizan recursos y se insertan cómodamente en el mercado agropecuario con una oferta de monoproducto. Sus rasgos empresariales no son sinónimo de grandes extensiones, lo cual contrasta con Jañez et al. (1990) y Radrizzani (2000), quienes asocian la categoría empresarial con grandes superficies. Los dos subconjuntos que lo componen se diferencian en la dimensión de sus explotaciones, lo cual indicaría que la superficie no es fundamental para distinguir la lógica de su funcionamiento; sus formas similares de gestión se explican más por la disponibilidad de los recursos trabajo y capital, por la realidad social y la orientación productiva que por el recurso tierra.

Grupo 2. Incluye $28 \%$ de la muestra y se caracteriza por:

- La superficie activa media es 164 ha.

- El nivel de instrucción es moderado alto; $37.5 \%$ es profesional y $37.5 \%$ tiene estudios secundarios completos. El tiempo promedio de experiencia rural es de 29 años.

- El grado de mecanización es alto en la mitad de los casos; $37.5 \%$ poseen grado 2 y el resto tiene grado 1. La potencia media de tracción es bastante alta (154 hp).

- Se contrata mano de obra extrapredial, pero ninguna explotación posee más de un empleado fijo. La ayuda de trabajo familiar es muy escasa.

\section{DeSarrollo}


- $56 \%$ de las explotaciones se dedica a la actividad mixta (agrícola y pecuaria) y $25 \%$ a la ganadería exclusivamente. Se produce maíz, algodón, anquito, sandía, cebolla y, en menor escala, batata y zanahoria. $62.5 \%$ de los productores consigna entre sus cultivos las pasturas forrajeras para la invernada de ganado vacuno.

- Poseen suelos de buena calidad, recuperados mediante buenas prácticas de manejo.

- $75 \%$ de los productores tiene ingresos extraagrarios.

La forma de gestión del grupo 2 se distingue del grupo anterior por la orientación y tamaño productivo y por sus características sociales. Es el grupo más numeroso, que corresponde a medianos productores con orientación ganadera, incorporados al mercado. El nivel de capitalización es alto en proporción al tamaño de sus fincas. Su dedicación parcial a la actividad está en relación con la obtención de ingresos extrapredio. Paz (2002) señala que, a mediados de la década de los noventa, surgieron en la zona nuevos productores que orientaron parte de su tiempo a la actividad ganadera.

Grupo 3. Comprende $17.5 \%$ de la muestra y lo forman productores que se caracterizan por:

- La superficie media en actividad es de 48 ha.

- El nivel de educación es el más bajo de todos los grupos; $60 \%$ tiene nivel primario y el resto no tiene instrucción. Es el grupo con más experiencia y trayectoria en la actividad rural; la edad promedio de antigüedad en la actividad es de 45 años.

- El nivel promedio de mecanización es muy bajo, con grado 1 en 50\% de los casos y grado 0 en el resto. La variable Рот (potencia media de tracción) presenta valores muy bajos: más de la mitad de los productores de este grupo no cuenta con tractores y el resto tiene una sola unidad de no más de $65 \mathrm{hp}$ de potencia.

- Ningún productor contrata empleados fijos y casi todos ocupan mano de obra familiar. El agricultor trabaja a tiempo completo con ayuda familiar.

- $80 \%$ reside en la explotación, como era de esperar.

- En 70\% de los casos se consignan como explotaciones con actividad mixta (agrícolaganadera); no obstante, la gran mayoría emprende pequeñas extensiones agrícolas diversificadas con el cultivo de sandía, anquito, maíz y alfalfa para fardos. La actividad pecuaria se limita a la cría de ganado menor (cerdos, gallinas y cabras) con muy poco de ganado vacuno.

- La calidad del suelo no es uniforme: $50 \%$ de los casos son de buena calidad y 50\% son de calidad regular.

- Los ingresos del predio son muy bajos. $60 \%$ declara no tener ingresos extras; $40 \%$ restante recibe algún tipo de transferencia formal (jubilación o pensión), que logra completar el nivel de subsistencia.

\section{DeSarrollo}


Este conglomerado está conformado por un reducido número de productores típicos de la zona de riego de la década de los años setenta (productor familiar tipo farmer), con producción diversificada, que subsiste pero con deteriorado nivel de actividad. Es el grupo de mayor experiencia, residente en la zona rural, con muy escasa disponibilidad de los recursos tierra, trabajo y capital, y con una situación social menoscabada.

Grupo 4. Representan $42 \%$ de la muestra y está integrado por productores con las siguientes características:

- La superficie activa promedio es de 72 ha. $83 \%$ de los miembros de este grupo tiene hasta 100 ha y $92 \%$ tiene hasta 200 ha.

- El nivel de educación es bajo. $62.5 \%$ posee instrucción primaria y $37.5 \%$ no tiene los estudios básicos. La antigüedad promedio en la actividad rural es de 39 años.

- El grado de mecanización es bastante aceptable: dos tercios del grupo exhibe grado 3 y el tercio restante, 2. La potencia media de tracción es elevada (168hp).

- Una gran mayoría (84\%) contrata mano de obra transitoria y solamente $29 \%$ posee en nómina a empleados fijos. Se consigna la ayuda de trabajo familiar.

- Se dedican exclusivamente a la actividad agrícola. Podría distinguirse tres subgrupos: el algodonero (40\%), el que cultiva maíz y trigo (representa 30\%), y 30\% restante cultiva cebolla, tomate para industria, sandía y anquito, entre otros productos.

- $54 \%$ señala que la calidad de sus suelos es buena.

- Los ingresos agrarios muestran un nivel intermedio entre los grupos 1 y 2.

El grupo 4 es el conjunto mayoritario de medianos productores, con experiencia rural, que genera puestos de trabajo transitorio y que se inserta al mercado agrícola con los productos tradicionales de esta zona de regadío (alfalfa y hortalizas). El grado de mecanización es notable, pero la maquinaria es obsoleta, que no han logrado renovar.

Con excepción del grupo 2, los otros conglomerados presentan gran uniformidad dentro de su tipo en cuanto a recursos disponibles, a las lógicas de producción y al origen de la similitud de los actores sociales que lo componen.

\section{Conclusiones}

La evaluación económica de la realidad de los productores agrarios permitió perfilar un diagnóstico actualizado para el área de riego del río Dulce, cuyo resumen es el siguiente:

- $52 \%$ de las explotaciones seleccionadas se encuentran actualmente improductivas. Este dato, contrastado con investigaciones anteriores, revela un proceso acelerado de abandono de la producción en el área.

\section{DeSarrollo}


- La zona despliega su actividad rural sobre la base de la agricultura, ya que $60 \%$ de los productores encuestados son exclusivamente agricultores.

- Desde la óptica socioeconómica del productor, se puede señalar que $44 \%$ de los jefes de explotación llevan entre 40 y 50 años al frente de la misma. Solamente $47 \%$ reside en la explotación rural; el resto se traslada diariamente desde un centro urbano cercano. Dos tercios de los agricultores no poseen estudios o sólo completaron la escuela primaria.

- $47 \%$ de los agricultores cubre sus necesidades económicas con la renta que se genera exclusivamente en la actividad rural; 53\% restante obtiene ingresos adicionales provenientes de transferencias formales, como jubilaciones o pensiones, o de otras actividades económicas, como la industria agropecuaria y el comercio.

Los resultados obtenidos del agrupamiento permitieron identificar cuatro explotaciones representativas o tipo del área de riego del río Dulce. Cada explotación tipo quedó definida por las características de su grupo de referencia, que conducen a formas de gestión rural bien diferenciadas entre sí:

- Explotación tipo 1: constituida por unas 600 ha encuadradas en un sistema productivo exclusivamente agrícola, con mano de obra permanente, un parque de maquinaria moderno y completo y sin restricciones serias de capital. El propietario tiene pocos años de experiencia en la actividad en comparación con las otras y se dedica a ésta a tiempo completo; consecuentemente, no tiene ingresos extras. El manejo actual de este tipo de explotación se identifica por laescasa diversificación productiva en grandes extensiones.

- Explotación tipo 2: de 160 ha, destinadas a la agricultura y ganadería, donde las tareas rurales están totalmente mecanizadas; con pocas restricciones de capital y mano de obra permanente. El productor realiza la supervisión y dirección del campo, pero utiliza parte de su tiempo en otras actividades que le generan ingresos extra.

- Explotación tipo 3: posee unas 48 ha con suelos de calidad regular, dedicadas totalmente a actividades agrícolas. Cuenta con un tractor e implementos básicos de arrastre con alto grado de obsolescencia. La producción está organizada con base en el trabajo del grupo doméstico, con un alto componente de labores manuales. El productor reside en el predio, tiene aproximadamente 45 años de experiencia en la actividad y no posee ingresos agrarios extra.

- Explotación tipo 4: tiene una extensión de 72 ha, con suelos de buena calidad, ocupadas para cultivos agrícolas monoespecíficos, como el algodón o una combinación de maíz y trigo. No hay mano de obra permanente y la fuerza laboral está formada por el propietario, un familiar y mano de obra eventual. Dispone de tractores e implementos de labranza, todos amortizados. La antigüedad del parque de maquinarias demuestra la imposibilidad de capitalización.

\section{DeSarrollo}


La tipificación obtenida permite detectar debilidades y fortalezas que contribuyen a establecer prioridades a la hora de diseñar políticas de desarrollo económico para la zona:

- Para aquellas explotaciones que se encuentran en una situación de fragilidad, se sugiere aprovechar su estructura de equipamiento, mecanización y acceso a la tierra para incrementar la productividad de sus sistemas con estrategias de producción múltiple que puedan optimar las condiciones de mercados cambiantes. Para ellas, las políticas deberían orientarse, fundamentalmente, a resolver las restricciones de la comercialización de sus productos por intermedio de asociaciones de productores y sistemas organizados de comercialización y de acciones articuladas entre los productores, las organizaciones intermedias y los gobiernos. Estas explotaciones pueden identificarse con los tipos 3 y 4, asociados a una agricultura de regadío en el límite de la extensión de la tierra y con cultivos de alto valor unitario. En particular, la explotación 3 es la más comprometida con el futuro del área, porque contiene al productor residente, con antigüedad y trayectoria en la actividad.

- Para los otros tipos de explotaciones de carácter expansionista, que poseen atributos de mayor sostenibilidad y que ostentan un buen nivel de administración, las acciones prioritarias tendrían que dirigirse a brindar apoyo con información técnica y económica que les sirva para encauzar el uso racional del agua de riego y del suelo. Las explotaciones tipo 1 y 2 encuadrarían en estos casos. Se perciben en ellas los intentos por introducir cultivos exportables (maíz, trigo, soja, algodón) que dinamizan la actividad rural. Estas explotaciones parecen presentar mayor capacidad de adecuación a la coyuntura, por medio de respuestas económicas y tecnológicas dentro de la actividad tradicional o incorporando nuevos productos, entre los que se destaca la ganadería vacuna de engorde y el cultivo de granos.

Por último, es importante subrayar que los resultados obtenidos en este trabajo para la zona de Santiago del Estero son extrapolables a otras zonas de regadío argentinas con situaciones similares. El eje de atención de la política agraria del país ha sido, históricamente, la agricultura pampeana, una actividad de secano. Por el contrario, la agricultura irrigada, actividad extrapampeana, tiene su centro de gravedad en productos regionales. Por tanto, las acciones de intervención pública que se derivan para la región en estudio pueden constituir la base de una futura política agraria con mayor apoyo al medio rural. 


\section{Bibliografía}

Afifi, A. A. y V. Clark, Computer-Aided Multivariate Analysis. Texts in Statistical Science, Boca Raton, Londres, Nueva York, Washington, Chapman y Hall CRC, 1999

Álvarez, R. y R. Paz, "Metodología asociada al diseño de propuestas para el desarrollo de la producción lechera caprina", en Archivos de Zootecnia, vol. 47, núm. 175, Ediciones Instituto de Zootecnia, Facultad de Veterinaria, Universidad de Córdoba, 1997.

-, "Lechería caprina y reconversión en los sistemas de producción campesinos", en Sistemas productivos campesinos, Santiago del Estero, Ediciones CICYT-UNSE, 1998.

Berdegué, J.; O. Sotomayor y C. Silleruelo, "Metodología de tipificación y clasificación de sistemas de producción campesinos de la provincia de Nuble, Chile", en Tipificación de sistemas de producción agrícola, Santiago de Chile, RIMISP, 1990, pp. 85-118.

CFI-DGEYC, Consejo Federal de Inversiones y Dirección General de Estadística y Censos, Producto Bruto Geográfico de Santiago del Estero. Período 1986-1997, Gobierno de la Provincia de Santiago del Estero, 1998.

Coronel de Renolfi, M. y S. Ortuño, “Optimización productiva en el área de riego del río Dulce de Santiago del Estero", en Realidad Económica núm. 203, Buenos Aires, Instituto Argentino para el Desarrollo Económico (IADE), 2004, pp. 121-136.

Cochran, W., Técnicas de muestreo, México, Compañía Editorial Continental, 1990

De Olivera Bassab; W.E. Shizue Miazaki y D. De Andrade, "Introdução à analise de agrupamentos", Associação Brasileira de Estadística, $9^{\circ}$ Simposio Nacional de Probabilidade e Estadística, São Paulo, 1990.

Escobar, G. y J. Berdegué, “Conceptos y metodología para la tipificación de sistemas de fincas: la experiencia de RIMISP", en Tipificación de sistemas de producción agrícola, Santiago de Chile, RIMISP, 1990, pp. 13-44

González García, C.; J. Martínez Falero; M. Pardo Méndez y J. Solana Gutiérrez, Técnicas de muestreo en la evaluación de recursos forestales, Madrid, Escuela Técnica Superior de Ingenieros de Montes, 1993.

Hart, R., "Componentes, subsistemas y propiedades del sistema finca como base para un método de clasificación", en Tipificación de sistemas de producción agrícola, Santiago de Chile, RIMISP, 1990, pp.45-62.
Jañez, H. y J.C. Antuña, Estudio de mercado de la alfalfa, Consejo Federal de Inversiones, Santiago del Estero, 2001.

Jañez, H., G. Sempronii y H. Neme, Caracterización del Sector Agropecuario de Santiago del Estero. Estudio para la implementación de la reforma impositiva agropecuaria, Proyecto PNUD Argentina 85/019, Subsecretaría de Agricultura, Ganadería y Pesca de la Nación, 1990.

Landín, R., "Tipificación de entidades geográficas y administrativas para priorizar zonas objetivo de proyectos de investigación agropecuaria", en Tipificación de sistemas de producción agrícola, Santiago de Chile, RIMISP, 1990, pp. 141-156.

Ledesma, N., "Caracteres de la semiaridez en el Chaco Seco", en El ambiente del Chaco Semiárido, Reunión Interacadémica, Academia Nacional de Agronomía y Veterinaria, Buenos Aires, 1992, pp. 21-44.

Manly, B., Multivariate Statistical Methods, Nueva York, Chapman y Hall, 1986.

Montenegro, C., El análisis multivariante en el diseño de modelos de simulación para los sistemas de producción campesinos, Universidad Católica de Santiago del Estero, 2000.

Paz, R., Sistemas de producción caprino en Santiago del Estero. Proyecciones y desafíos para el desarrollo del sector, Santiago del Estero, Fundapaz, 2002.

Radrizzani, A., Los sistemas productivos del área de riego del río Dulce. Una visión desde la sustentabilidad, Universidad Internacional de Andalucía, 2000.

Rodríguez Ocaña, A; J. Berbel Vecino y P. Ruiz Avilés, Metodología para el análisis de la toma de decisiones de los agricultores, Monografías INIA, núm. 101, Madrid, Instituto Nacional de Investigación y Tecnología Agraria y Alimentaria, Ministerio de Agricultura, Pesca y Alimentación, 1998.

Scheaffer, R., W. Mendenhall y L. Ott, Elementos de muestreo, México, Iberoamérica, 1987.

Suárez, R. y L. Escobar, "Tipificación de fincas en la comarca de Fusagasuga, Colombia, según sus tendencias de cambio técnico", en Tipificación de sistemas de producción agrícola, Santiago de Chile, RIMISP, 1990, pp. 181-200.

UESRRD, Unidad Ejecutora del Servicio de Riego del río Dulce: Informe general, Ministerio de Economía, Gobierno de la Provincia de Santiago del Estero, 1992. 